\title{
Effects of Xiao-Ban-Xia-Tang Formula on Cisplatin and 1- phenylbiguanide Hydrochloride Induced Emesis and Gut Microbiota in the Pica Model of Rats
}

\section{Yanhong Yang}

The First Affiliated Hospital (School of Clinical Medicine), Guangdong Pharmaceutical University, Nong-Lin-Xia Road 19\#, YueXiu District, Guangzhou 510080, P.R. China https://orcid.org/0000-0002-3923-5523

\section{Xiaodi Feng}

School of Chinese Materia Medica, Guangdong Pharmaceutical University, Guangzhou Higher Education Mega Center, Guangzhou, 510006, P.R.China

\section{Siqi Chen}

School of Chinese Materia Medica, Guangdong Pharmaceutical University, Guangzhou Higher Education Mega Center, Guangzhou, 510006, P.R.China

\section{Qi Meng}

School of Chinese Materia Medica, Guangdong Pharmaceutical University, Guangzhou Higher Education Mega Center, Guangzhou, 510006, P.R.China

\section{Qianqian Cheng}

School of Chinese Materia Medica, Guangdong Pharmaceutical University, Guangzhou Higher Education Mega Center, Guangzhou, 510006, P.R.China

Ke Nie ( $\nabla$ nicknk@hotmail.com )

School of Chinese Materia Medica, Guangdong Pharmaceutical University, Guangzhou Higher Education Mega Center, Guangzhou, 510006, P.R.China https://orcid.org/0000-0003-4410-7662

\section{Research}

Keywords: Xiao-Ban-Xia-Tang, pica, gut microbiota, cisplatin, 1-phenylbiguanide hydrochloride, ondansetron

Posted Date: August 11th, 2020

DOI: https://doi.org/10.21203/rs.3.rs-53216/v1

License: @ (i) This work is licensed under a Creative Commons Attribution 4.0 International License. Read Full License 


\section{Abstract}

Background: In this study, the effects of Xiao-Ban-Xia-Tang (XBXT) formula on cisplatin and 1-phenylbiguanide hydrochloride (1-PBG) induced acute and delayed emesis and gut microbiota were studied in the pica model of rats, compared with ondansetron.

Methods: Two rat models of cisplatin and 1-PBG induced pica were established, the amount of kaolin intake was observed, and the effects of XBXT and ondansetron on the gut microbiota were further studied by 16S rDNA gene analysis.

Results: The results showed that the total intake of kaolin of the rats injected with cisplatin and 1-PBG was significantly increased, and treatment of XBXT and ondansetron could significantly ameliorate the acute and delayed pica induced by cisplatin and 1-PBG. The 16S rDNA gene analysis has shown that the alpha diversity of the gut microbiota of the cisplatin and 1-PBG treated rats was significantly decreased compared with the control group, and ondansetron could further decrease the alpha diversity of the gut microbiota of the rats treated with cisplatin. Ondansetron significantly decreased the relative abundance of Firmicutes and increased the abundance of Bacteroidetes on phylum level in the cisplatin and 1-PBG treated rats, while XBXT only decreased Firmicutes in the cisplatin treated rats.

Conclusions: XBXT was as effective as ondansetron in the treatment of acute and delayed pica induced by cisplatin and 1-PBG in rats. Ondansetron was more likely to cause gut microbiota dysbiosis than XBXT. Our study provided new avenues for the roles and mechanisms of XBXT on the prevention and treatment of CINV.

\section{Background}

Nausea and vomiting is one of the most common and painful symptoms in humans. Gastrointestinal disease, endocrine or metabolic diseases, surgery, neurological or psychiatric factors, pregnancy and some medicines can cause nausea and vomiting. Chemotherapy-induced nausea and vomiting (CINV) is one of the severe adverse effects of chemotherapy, which can affect the quality of life and compliance of cancer patients. CINV can occur in the acute phase $(0-24 \mathrm{~h})$ and in the delayed phase (25-120 h) after chemotherapy, and many neuroreceptors such as dopamine, serotonin, and neurokinin-1 (NK1), have been found to be involved in the pathological process of chemotherapeutic vomiting $[1,2]$. The main medicines used for the treatment of CINV include 5-Hydroxytryptamine (5-HT3) receptor antagonists, NK1 receptor antagonists, corticosteroids, dopamine antagonists, benzodiazepines, cannabinoids and olanzapine [3]. These medicines usually act on a single target, and are often needed to be combined with other medicines for the treatment of CINV except for expensive costs and side effects, such as constipation, headache and hiccups, et al [4]. In addition, it has been reported that delayed CINV has a higher incidence and is less responsive to treatment compared with acute CINV $[1,2]$. So it is still urgent to find potential medicines for the prevention and treatment of acute and delayed CINV.

Traditional medicine converts traditional theories and experiences into medical knowledge and skills to prevent and treat diseases, and has been recommended by the World Health Organization as complementary and alternative medicine for its effect, safety, availability and low cost [5]. Xiao-Ban-Xia-Tang (XBXT) formula, a traditional Chinese medicine recipe, has been used in China for more than 2000 years. XBXT includes only two herbal medicines, ginger (Zingiber officinale Roscoe) and pinellia (Pinellia ternata ( Thunb.) Breit.), and is usually used for the treatment of vomiting [6]. Ginger and its extracts have been found to have many biological and pharmaceutical activities, such as anti-inflammatory, anticancer, antiobesity, antinausea and antiemetic activities [7]. Our previous study has shown that [6]-Gingerol, a major component of ginger, could significantly ameliorate the cisplatin-induced pica in rats [8]. Pinellia species also have many biological activities including antiinflammatory, anticancer, insecticidal, antitussive, anticonvulsant and antiemetic activities [9]. The main active components of Pinellia include alkaloids, lectins, fatty acids, cerebrosides, phenylpropanoids, sterols and flavonoids [9. 10]. Qian Q et al reported that XBXT could ameliorate cisplatin-induced emesis in minks by inhibiting the increase of Neurokinin-1 receptor (NK1R) [6]. Qian Q's another study reported that XBXT could improve cisplatin-induced pica in rats by inhibiting the increase of central or peripheral obestatin and blood Cholecystokinin (CCK) and Calcitonin gene-related peptide (CGRP) [11]. Since 
traditional Chinese medicine recipe usually have many ingredients and multi-targets, the mechanism of XBXT remain not clear yet.

In our previous study, the effects of ondansetron and [6]-gingerol on pica and gut microbiota in rats injected with cisplatin were evaluated [8]. In the present study, two nausea and vomiting models of rats have been established: one was induced by cisplatin, and the other was induced by 1-phenylbiguanide hydrochloride (1-PBG), which is an agonist of 5HT3 [12]. The pica behavior, consumption of kaolin (china clay), was used as an indicator of nausea and vomiting. Both cisplatin and 1-PBG caused acute and delayed behavior of pica. Ondansetron and XBXT were used to treat the two models of rats, and the effects of Ondansetron and XBXT on the gut microbiota of the cisplatin and 1-PBG treated rats were further checked by 16S rDNA gene analysis, providing new sights for the prevention and treatment of CINV.

\section{Materials And Methods}

\section{Preparation of kaolin pellets}

The preparation of kaolin pellets was described in our previous work [8].

\section{Preparation of XBXT}

Ginger (Zingiber officinale Roscoe, produced in Laiwu, Shandong Province, China, voucher specimen no. J7201) and pinellia (Pinellia ternata (Thunb.) Breit., produced in Xihe County, Gansu Province China, voucher specimen no.J7654) were identified by Professor Jizhu Liu from School of Chinese Materia Medica, Guangdong Pharmaceutical University. Voucher specimens were deposited at the Herbarium of Traditional Chinese Medicine of Guangdong Pharmaceutical University.

Ginger $200 \mathrm{~g}$ and pinellia $400 \mathrm{~g}$ were selected and weighed precisely. The herbs were soaked in $4800 \mathrm{~mL}$ distilled water overnight, and back-flowed for $1.5 \mathrm{~h}$. The extract was filtered, and the residual medicine was soaked in distilled water following the same procedure once more. The pool of the extracts from two soaking and filtering was lyophilized to form a dried powder.

The quality of XBXT was further checked by high performance liquid chromatography (HPLC), and [6]-gingerol, [6]-shogaol, ephedrine and succinic acid were used for quality control. Pure standards of [6]-gingerol (Manst Biotechnology Co., Ltd. Chendu, China), [6]-shogaol (Manst Biotechnology Co., Ltd. Chendu, China), ephedrine (China Institute of Food and Drug Verification) and succinic acid (China Institute of Food and Drug Verification) were used as external standards of the HPLC analysis. For the check of [6]-gingerol and [6]-shogaol, $0.3 \mathrm{~g}$ dried powder of XBXT was dissolved in $1 \mathrm{~mL}$ petroleum ether, and filtered through a $0.45 \mu \mathrm{m}$ filter (Jinteng Experimental Equipment Co., Ltd., Tianjin, China). The HPLC analysis was performed using the HPLC system (U3000, Thermo Fisher Scientific) with acetonitrile as mobile phase A and $0.1 \%$ formic acid solution as mobile phase B at $280 \mathrm{~nm}$. The flow rate was $0.5 \mathrm{~mL} / \mathrm{min}$ and the column temperature was $30{ }^{\circ} \mathrm{C}$. For the check of ephedrine, $0.3 \mathrm{~g} \mathrm{XBXT}$ powder was dissolved in $1 \mathrm{~mL} 75 \%$ ethanol (containing 1\% hydrochloric acid). The mobile phase was methanol: $0.08 \%$ triethylamine aqueous solution $(\mathrm{pH}=5.8)$. The flow rate was $1.0 \mathrm{~mL} /$ minute and the detection wavelength was $210 \mathrm{~nm}$. For the check of succinic acid, $0.3 \mathrm{~g}$ XBXT powder was dissolved in $1 \mathrm{~mL}$ petroleum ether. The mobile phase was methanol: $0.02 \mathrm{~mol} / \mathrm{L}$ $\mathrm{KH}_{2} \mathrm{PO}_{4}$ buffer $(\mathrm{pH}=2.5)$. The flow rate was $1.0 \mathrm{~mL} /$ minute and the detection wavelength was $214 \mathrm{~nm}$. Petroleum ether, acetonitrile, formic acid, ethanol, hydrochloric acid, methanol, triethylamine and $\mathrm{KH}_{2} \mathrm{PO}_{4}$ were purchased from Zhiyuan Chemical Reagent Co., Ltd., Tianjin, China. The results of HPLC analysis were shown in Figure S1-3.

\section{Animal treatment}

All the animal experiments were approved by the Committee on Laboratory Animal Care and Use of Guangdong Pharmaceutical University (Guangzhou, China), in accordance with the National Institutes of Health guide for the care and use of laboratory animals. The Wistar rats (180-220g) (provided by Peng Yue experimental animal Co., Ltd., Jinan, China) were housed in a temperature-controlled room $\left(25^{\circ} \mathrm{C}\right)$, and the relative humidity was $40 \%-60 \%$.

The kaolin pellets were introduced into the rats 3 days prior to drug administration. Most of the rats did not take kaolin anymore at the third day, and the rats that were still interested in kaolin were excluded. The rest rats were randomly divided into seven 
groups and each group had 6 rats. Group 1 was the normal control group (Control); group 2 was the rats treated with cisplatin (C-Model); group 3 was the rats treated with cisplatin and ondansetron (C-Ond); group 4 was the rats treated with cisplatin and the XBXT (C-XBXT); group 5 was the rats treated with 1-PBG (P-Model); group 6 was the rats treated with 1-PBG and ondansetron (P-Ond); group 7 was the rats treated with 1-PBG and XBXT (P-XBXT).

On the day of drug administration, the C-Ond group and the P-Ond group were given ondansetron ( $1.3 \mathrm{mg} / \mathrm{kg}$ (body weight), Qilu Pharmaceutical Company, China) by gavage respectively. The C-XBXT group and the P-XBXT group were given XBXT (0.16 $\mathrm{g} / \mathrm{mL}$ ) by gavage respectively. The control group, the C-Model group and the P-Model group were given pure water by gavage. After $1 \mathrm{~h}$, the C-Model group, C-Ond group and the C-XBXT group were injected intraperitoneally (i.p.) with cisplatin (Qilu Pharmaceutical Company, China) at the concentration of $6 \mathrm{mg} / \mathrm{kg}$ (body weight); the P-Model group, P-Ond group and the PXBXT group were injected i.p. with 1-PBG (Sigma) at the concentration of $25 \mathrm{mg} / \mathrm{kg}$ (body weight), and the control group was injected i.p. with saline of equal volume. The $\mathrm{C}$-Ond group and the P-Ond group were continued to be given ondansetron (1.3 $\mathrm{mg} / \mathrm{kg}$ body weight) by gavage and the C-XBXT group and the P-XBXT group were continued to be given XBXT $(0.16 \mathrm{~g} / \mathrm{mL})$ by gavage, twice each day for three days. The general conditions, the body weight and kaolin intake of rats were measured every $24 \mathrm{~h}$, and recorded until $72 \mathrm{~h}$ after the establishment of the models.

\section{S rDNA gene analysis}

After the establishment of the models for $72 \mathrm{~h}$, fecal samples were collected. Fecal bacterial DNA extraction, 16S rDNA gene PCR amplification, sequencing and analysis were carried out by Gene Denovo Biotechnology Company (Guangzhou, China). The experimental procedures were performed as previously described $[8,13]$.

\section{Statistical analysis}

Statistical differences were determined by using SPSS 23.0 software (SPSS Inc., Chicago, IL, USA). Data were expressed as mean \pm S.E. Two-way ANOVA was performed when more than two groups were compared, $P$ value less than 0.05 was considered to be significant.

\section{Results}

\section{Effect of XBXT on cisplatin and 1-PBG induced kaolin intake}

All the rats took kaolin at the first day of kaolin release, and the amount of kaolin intake was gradually decreased at the following 2 days. At the third day, almost all rats did not take kaolin anymore. After injected i.p. of cisplatin, the total intake of kaolin in the $\mathrm{C}$-model group was significantly higher than the control group $(P<0.001)$. The amount of kaolin intake of the CModel group reached the peak at $24 \mathrm{~h}$, and the second higher amount appeared at $72 \mathrm{~h}$ (Fig. 1A and Table 1). After injected i.p. of 1-PBG, the total intake of kaolin in the P-model group was significantly higher than the control group at $24 \mathrm{~h}(P<0.001)$, and the amount reached the highest at $72 \mathrm{~h}$ (Fig. $1 \mathrm{~A}$ and Table 1). Both ondansetron and XBXT could significantly decrease the kaolin intake of the rats treated with cisplatin at $24 \mathrm{~h}$ and $72 \mathrm{~h}$, and for rats treated with 1-PBG, both ondansetron and XBXT could also obviously decrease the kaolin intake at $24 \mathrm{~h}$ and $72 \mathrm{~h}$ (Fig. 1A and Table 1). Figure 1B demonstrated the total amount of kaolin intake from $0 \mathrm{~h}$ (cisplatin and 1-PBG were injected i.p. at $0 \mathrm{~h}$ ) to $72 \mathrm{~h}$ of the 7 groups of rats, and the amount of kaolin intake was significantly increased in the $\mathrm{C}$-Model group and the P-Model group $(P<0.01$, Fig. 1B). Ondansetron could significantly inhibit the kaolin intake of the cisplatin and 1-PBG treated rats $(P<0.05$, Fig.1B), and XBXT could inhibit the kaolin intake of the 1-PBG treated rats $(P<0.01$, Fig. 1B).

After cisplatin and 1-PBG injection, the body weight of rats in the C-Model group and the C-XBXT group was significantly decreased compared with the control group $(P<0.001)$, and there was no significant difference of the body weight between other groups (Fig. 1C, Table 2).

\section{Overview of the $16 \mathrm{~S}$ rDNA gene analysis}


The effects of ondansetron and XBXT on the gut microbiota of cisplatin and 1-PBG treated rats were investigated by 16S rDNA gene sequencing. The total tags, operational taxonomic units (OTUs), the Ace index, Chao index, Shannon index and Simpson index were shown in Table 3. The Shannon rarefaction curves for each group have reached the saturation plateau, indicating that the samples had enough sequence coverage (Fig. 2A). The Venn diagram showed that there were 527 common OTUs in the 7 groups of rats (Fig. 2B), there were 597 common OTUs in the control group and the groups treated with cisplatin (Fig. 2C), and there were 637 common OTUs in the control group and the groups treated with 1-PBG (Fig. 2D).

\section{The effects of XBXDT on the gut microbiome of cisplatin and 1-PBG treated rats}

Figure 3A demonstrated the Chao index of the 7 groups of rats. The alpha diversity of the gut microbiota of the C-Model and PModel group was significantly decreased compared with the control group $(P<0.01$ and $P<0.001$ respectively), and ondansetron could further decrease the alpha diversity of the gut microbiota of the rats treated with cisplatin $(P<0.01, \mathrm{Fig} .3 \mathrm{~A})$. Figure 3B showed the Principal co-ordinates analysis (PCOA) of the 7 groups of rats.

Figure 4A-B demonstrated the relative abundance of gut microbiota on phylum level, and Bacteroidetes, Firmicutes, Verrucomicrobia and Proteobacteria had high abundance in all the samples. The abundance of Firmicutes had no obvious change in the C-Model group and the P-Model group compared with the control group, but was significantly decreased in the COnd, C-XBXT and P-Ond groups compared with the control group ( $P<0.001$, Fig. 4A-B). The abundance of Bacteroidetes of the COnd group and the P-Ond group was significantly increased compared with the control group ( $P<0.001$, Fig. 4A-B). The abundance of Verrucomicrobia of the C-Model group and the C-XBXT group was significantly increased compared with the control group ( $P<0.05$ for the C-Model group, $P<0.001$ for the C-XBXT group, Fig. 4A-B). The relative abundance of gut microbiota on class level was demonstrated in Figure S4. The abundance of Clostridia was significantly decreased in the CModel, C-Ond, C-XBXT, P-Model, P-Ond and P-XBXT groups compared with the control group (Fig. S4). The abundance of Bacteroidia of the C-Ond group and the P-Ond group was significantly increased compared with the control group (Fig. S4).

Figure 5A-5B demonstrated the LEFse analysis of the cisplatin treated groups. The specific and predominant bacteria of the control group included Clostridlales_vadinBB60_group, Lachnospiraceae_NK4A16_group, Angelakisella, Ruminiclostridium_6, Ruminococcaceae_UCG_014, Ruminococcus_2, Candidatus_Saccharimonas, Saccharimonadaceae, Saccharimonadales, Saccharimonadla, Mollicutes_RF39 and Mollicutes, and the specific bacteria of the C-Model group was Clostridlales_bacterium_CIEAF_020 (Fig. 5A-5B). Figure 5C-5D showed the specific and predominant bacteria of the 1-PBG treated rats. Clostridiales and Clostridia were specific for the control group; Candidatus_Saccharimonas, Saccharimonadaceae, Saccharimonadales and Saccharimonadia were specific for the P-Model group; Prevotella_9, Fusicatenibacter, Phascolarctobacterium, Acidaminococcaceae, Selenomonadales, Negativicutes, Parasutterella, Burkholderiaceae, Betaproteobacteriales and Gammaproteobacteria were predominant in the P-Ond group; Lactobacillus_reuteri, Lactobacillus, Lactobacillaceae, Lactobacillales and Bacilli were predominant in the P-XBXT group (Fig. 5C-5D).

\section{Discussion}

In the present study, rats were treated with cisplatin and 1-PBG, and the consumption of kaolin was checked as an indicator of nausea and vomiting. Both cisplatin and 1-PBG could induce acute and delayed emesis. The amount of kaolin intake of the CModel group reached the peak at $24 \mathrm{~h}$, while the amount of kaolin intake of the P-Model group reached the peak at $72 \mathrm{~h}$. It seemed that cisplatin mainly caused acute emesis, and 1-PBG mainly caused delayed emesis in rats. 5-HT3 receptor antagonists and dexamethasone are the standard anti-emetic regimen for patients receiving highly emetogenic forms of chemotherapy (HEC) such as cisplatin-based regimens; however, delayed emesis remains a problem, since only about half of the cancer patients can get complete control of emesis after chemotherapy [14]. 1-PBG is a 5HT3 agonist, and rats injected i.p. with 1-PBG were induced acute and delayed CINV, moreover, 1-PBG induced CINV mainly presented as delayed CINV. Therefore, 1-PBG provided a good animal model, which could be used for the study of delayed CINV in future.

Many neurotransmitters pathways are involved in the pathological process of CINV, and there is no single neurotransmitter responsible for all forms of CINV, therefore, the prevention and treatment of CINV need the combination of several agents [15]. Although the standard anti-emetic regimen of 5-HT3 receptor antagonists and dexamethasone has been used for many years in

Page 5/15 
patients receiving HEC [14], more and more medicine are being developed for the prevention and treatment of CINV. In the present study, the effects of XBXT, a famous traditional Chinese medicine formula for vomiting, on cisplatin and 1-PBG induced acute and delayed emesis and the gut microbiota were studied, compared with ondansetron, the typical medicine for CINV. Our results showed that both ondansetron and XBXT could significantly inhibit the kaolin intake induced by cisplatin and 1-PBG at $24 \mathrm{~h}$ and $72 \mathrm{~h}$. Moreover, for the 1-PBG rat model, the amount of kaolin intake of the XBXT group was even lower than the ondansetron group (Fig. 1A-B and Table 1), indicating that XBXT might be more effective in the prevention and treatment of $5 \mathrm{HT} 3$ involved CINV.

The gut microbiota has got great interest in recent years since it plays important roles in maintaining health and the development of many diseases $[16,17]$. Due to the complex composition and complicated mechanism of actions, the potential clinical application of herbal medicine has not been widely recognized yet [18]. With the advancements in genome sequencing technologies and bioinformatics, gut microbiota has become a new avenue to study the traditional medicine [19]. In the present study, the effects of XBXT on the gut microbiota of cisplatin and 1-PBG treated rats were further checked by 16S rDNA gene sequencing analysis, compared with ondansetron. The results of 16S rDNA gene analysis showed that the alpha diversity of the gut microbiota of the C-Model and P-Model group was significantly decreased compared with the control group, and ondansetron further decreased the alpha diversity of the gut microbiota of the rats treated with cisplatin. Feng $\mathrm{Y}$ et al. reported that Bifidobacterium and Lactobacillus were associated with alpha diversity, beta diversity, and the robustness of the gut microbiota, and people harboring Bifidobacterium present but no Lactobacillus showed higher alpha diversity and were more robust than those only carrying Lactobacillus [20]. Increased microbiome diversity could promote the stability of the microbiome, thereby beneficial to the host for resistance against extreme stress and perturbations [21]. In the present study, both cisplatin and 1-PBG could decrease the alpha diversity of the gut microbiota, while ondansetron could further significantly decrease the alpha diversity of the gut microbiota of the rats treated with cisplatin. XBXT did not further decrease the alpha diversity of the gut microbiota of the cisplatin treated rats, therefore, ondansetron might have more severe side effects than XBXT.

In the present study, the abundance of Firmicutes was significantly decreased in the C-Ond, C-XBXT and P-Ond groups compared with the control group, while the abundance of Bacteroidetes was significantly increased in the C-Ond group and the P-Ond group compared with the control group. Ondansetron significantly decreased the relative abundance of Firmicutes and increased the abundance of Bacteroidetes, which was consistent with the result of our previous study [8], while XBXT only decreased Firmicutes in the cisplatin treated rats. Zeng $\mathrm{Y}$ et al reported that the abundance of Firmicutes was decreased, and Bacteroidetes was increased in patients with chronic hepatitis, liver cirrhosis and hepatocellular carcinoma compared with the healthy controls [22]. Kim JW et al reported that the Firmicutes/Bacteroidetes ratio was consistently reduced in systemic lupus erythematosus (SLE) patients [23]. On the contrary, Qiu D et al reported that the bacterial communities of the glucocorticoidinduced obesity group of people were increased in Firmicutes and decreased in Bacteroidetes compared with the healthy controls [24]. Zhao L et al reported that the ratio of Firmicutes/ Bacteroidetes was higher in type 2 diabetes (T2D) patients than the healthy controls [25]. Therefore, considering the high diversity of gut microbiota, the unexpected microbiota-host relationships and the underlying molecular mechanisms remain to be explored, and food and drugs can profoundly affect the gut bacterial community and change the activities of the gut microbiota [26]. The question "does an altered gut microbiota contribute to disease, or does it merely reflect a disease status?" remains not solved yet [27]. From the results of the present study, it seemed that ondansetron was more likely to cause gut microbiota dysbiosis than XBXT. An X et al reported that the interactions between the gut microbiota and herbal medicines occur mainly by two pathways: one is that the gut microbiota disintegrates the herbal medicines into metabolites and signaling molecules, which induce the physiological changes of the body; the other is that herbal medicines regulate the composition and relative abundance of the gut microbiota, and induce physiological changes [18]. The effects of XBXT on the gut microbiota and the underlying mechanisms still need further study.

\section{Conclusion}

In summary, ondansetron and XBXT could significantly ameliorate the acute and delayed pica induced by cisplatin and 1-PBG in rats. The alpha diversity of the gut microbiota of the C-Model and P-Model group was significantly decreased compared with the control group, and ondansetron could further decrease the alpha diversity of the gut microbiota of the rats treated with cisplatin.

Page 6/15 
Ondansetron significantly decreased the relative abundance of Firmicutes and increased the abundance of Bacteroidetes in the cisplatin and 1-PBG treated rats, while XBXT only decreased Firmicutes in the cisplatin treated rats. Ondansetron was more likely to cause gut microbiota dysbiosis than XBXT. XBXT could be used for the prevention and treatment of CINV, open new avenues to the mechanisms of herbal medicines associated with the gut microbiota.

\section{Abbreviations}

XBXT: Xiao-Ban-Xia-Tang; 1-PBG: 1-phenylbiguanide hydrochloride; CINV: chemotherapy-induced nausea and vomiting; NK1: neurokinin-1; 5-HT3: 5-Hydroxytryptamine; SLE: systemic lupus erythematosus; T2D: type 2 diabetes.

\section{Declarations}

\section{Ethics approval and consent to participate}

All the animal experiments were approved by the Committee on Laboratory Animal Care and Use of Guangdong Pharmaceutical University (Guangzhou, China).

\section{Consent for publication}

All authors agree with the submission.

\section{Availability of data and material}

The data of 16S rDNA gene analysis have been uploaded to figshare (https://figshare.com/),

https://figshare.com/articles/Effects_of_Xiao-Ban-Xia-Tang_formula_on_cisplatin_and_1-

phenylbiguanide_hydrochloride_induced_acute_and_delayed_emesis_and_gut_microbiota_in_the_pica_model_of_rats/11316230

Anyone who is interested in the study of gut microbiota, chemotherapy-induced nausea and vomiting, and related fields can download the data for free.

\section{Competing interests}

The author reports no conflicts of interest in this work.

\section{Funding}

This work was supported by the National Natural Science Foundation of China (No. 81673779, No.81803912), the Scientific Research Project of the Administration of Traditional Chinese Medicine of Guangdong Province (No. 20182079), the Characteristic Innovation Project (Natural Science) of the Education Department of Guangdong Province and the "Innovation Strong School Project" of Guangdong Pharmaceutical University (No.2017KTSCX102), and the Science and Technology Project of Yue-Xiu District of Guangzhou (No. 2018-WS-011).

\section{Author Contributions}

Ke Nie designed the study and conceived the report. Yanhong Yang conceived the report, analyzed and interpreted the results, wrote the first draft of the manuscript and revised it critically. Xiaodi Feng, Siqi Chen, Qi Meng and Qianqian Cheng performed the experiments. Xiaodi Feng created the figures.

\section{Acknowledgements}

Not applicable.

\section{References}


1. Karthaus M, Schiel X, Ruhlmann CH, Celio L. Neurokinin-1 receptor antagonists: review of their role for the prevention of chemotherapy-induced nausea and vomiting in adults. Expert Rev Clin Pharmacol. 2019; 12:661-80.

2. Schwartzberg L, Karthaus M, Rossi G, Rizzi G, Borroni ME, Rugo HS, et al. Fixed combination of oral NEPA (netupitantpalonosetron) for the prevention of acute and delayed chemotherapy-induced nausea and vomiting in patients receiving multiple cycles of chemotherapy: Efficacy data from 2 randomized, double-blind phase III studies. Cancer Med. 2019; 8:2064-73.

3. Adel N. Overview of chemotherapy-induced nausea and vomiting and evidence-based therapies. Am J Manag Care. 2017; 23:S259-65.

4. Hu J, Shen Y, Zhang G, He J, Sun M, Zhang H, et al. Effect of acupoint therapies on chemotherapy-induced nausea and vomiting: A systematic review protocol. Medicine (Baltimore). 2019; 98:e17109.

5. Ortiz LM, Lombardi P, Tillhon M, Scovassi Al. Berberine, an epiphany against cancer. Molecules. 2014; 19:12349-67.

6. Qian Q, Chen W, Yue W, Yang Z, Liu Z, Qian W. Antiemetic effect of Xiao-Ban-Xia-Tang, a Chinese medicinal herb recipe, on cisplatin-induced acute and delayed emesis in minks. J Ethnopharmacol. 2010; 128:590-3.

7. Mao QQ, Xu XY, Cao SY, Gan RY, Corke H, Beta T, et al. Bioactive Compounds and Bioactivities of Ginger (Zingiber officinale Roscoe). Foods. 2019; 8. pii: E185.

8. Feng X, Cheng Q, Meng Q, Yang Y, Nie K. Effects of ondansetron and [6]-gingerol on pica and gut microbiota in rats treated with cisplatin. Drug Des Devel Ther. 2019; 13: 2633-41.

9. Ji X, Huang B, Wang G, Zhang C. The ethnobotanical, phytochemical and pharmacological profile of the genus Pinellia. Fitoterapia. 2014; 93:1-17.

10. Jie EY, Ryu YB, Choi SA, Ahn MS, Liu JR, Min SR, et al. Mass propagation of microtubers from suspension cultures of Pinellia ternatacells and quantitative analysis of succinic acid in Pinellia tubers. Plant Biotechnol Rep. 2015; 9:331-8.

11. Qian Q, Chen W, Guo C, Wu W, Qian W, Li S. Xiao-Ban-Xia-Tang inhibits cisplatin-induced pica by down regulating obestatin in rats. J Ethnopharmacol. 2011; 135:186-93.

12. Ataee R, Ajdary S, Rezayat M, Shokrgozar MA, Shahriari S, Zarrindast MR. Study of 5HT3 and HT4 receptor expression in HT29 cell line and human colon adenocarcinoma tissues. Arch Iran Med. 2010; 13:120-5.

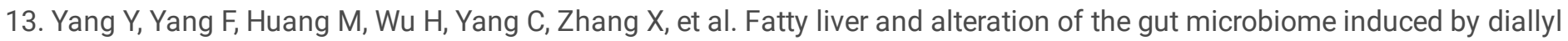
disulfide. Int J Mol Med. 2019; 44: 1908-20.

14. Hu W, Fang J, Nie J, Dai L, Chen X, Zhang J, et al. Addition of aprepitant improves protection against cisplatin-induced emesis when a conventional anti-emetic regimen fails. Cancer Chemother Pharmacol. 2014; 73:1129-36.

15. Celio L, Ricchini F, De Braud F. Safety, efficacy, and patient acceptability of single-dose fosaprepitant regimen for the prevention of chemotherapy-induced nausea and vomiting. Patient Prefer Adherence. 2013; 7:391-400.

16. Sánchez-Tapia M, Tovar AR, Torres N. Diet as regulator of gut microbiota and its role in health and disease. Arch Med Res. 2019; 50:259-68.

17. Tilg H, Cani PD, Mayer EA. Gut microbiome and liver diseases. Gut. 2016; 65:2035-44.

18. An X, Bao Q, Di S, Zhao Y, Zhao S, Zhang H, et al. The interaction between the gut microbiota and herbal medicines. Biomed Pharmacother. 2019; 118:109252.

19. Yue SJ, Wang WX, Yu JG, Chen YY, Shi XQ, Yan D, et al. Gut microbiota modulation with traditional Chinese medicine: A system biology-driven approach. Pharmacol Res. 2019; 104453. doi: 10.1016/j.phrs.2019.104453.

20. Feng Y, Duan Y, Xu Z, Lyu N, Liu F, Liang S, et al. An examination of data from the American Gut Project reveals that the dominance of the genus Bifidobacterium is associated with the diversity and robustness of the gut microbiota. Microbiologyopen. 2019; e939. doi: 10.1002/mbo3.939.

21. Dhillon J, Li Z, Ortiz RM. Almond snacking for 8 wk increases alpha-diversity of the gastrointestinal microbiome and decreases bacteroides fragilis abundance compared with an isocaloric snack in college freshmen. Curr Dev Nutr. 2019; 3:nzz079. 
22. Zeng Y, Chen S, Fu Y, Wu W, Chen T, Chen J, et al. Gut microbiota dysbiosis in patients with hepatitis B virus-induced chronic liver disease covering chronic hepatitis, liver cirrhosis and hepatocellular carcinoma. J Viral Hepat. 2019; doi:

10.1111/jvh.13216.

23. Kim JW, Kwok SK, Choe JY, Park SH. Recent advances in our understanding of the link between the intestinal microbiota and systemic lupus erythematosus. Int J Mol Sci. 2019; 20. pii: E4871.

24. Qiu D, Xia Z, Deng J, Jiao X, Liu L, Li J. Glucorticoid-induced obesity individuals have distinct signatures of the gut microbiome. Biofactors. 2019; doi: 10.1002/biof.1565.

25. Zhao L, Lou H, Peng Y, Chen S, Zhang Y, Li X. Comprehensive relationships between gut microbiome and faecal metabolome in individuals with type 2 diabetes and its complications. Endocrine. 2019; doi: 10.1007/s12020-019-02103-8.

26. Lee WJ, Hase K. Gut microbiota-generated metabolites in animal health and disease. Nat Chem Biol. 2014; 10:416-24.

27. Schroeder BO, Bäckhed F. Signals from the gut microbiota to distant organs in physiology and disease. Nat Med. 2016; 22:1079-89.

\section{Tables}

Table 1. Effects of XBXT on kaolin consumption in pica model rats $\square \pm S E M \square g \square$

\begin{tabular}{|lllllll|}
\hline & $-48 \mathrm{~h}$ & $-24 \mathrm{~h}$ & 0 & $24 \mathrm{~h}$ & $48 \mathrm{~h}$ & $72 \mathrm{~h}$ \\
\hline Control & $0.770 \pm 0.432$ & $0.274 \pm 0.097$ & $0.017 \pm 0.013$ & $0.066 \pm 0.028$ & $0.055 \pm 0.033$ & $0.154 \pm 0.048$ \\
\hline C-Model & $0.172 \pm 0.074$ & $0.148 \pm 0.113$ & $0.031 \pm 0.016$ & $1.383 \pm 0.181^{\star \star \star}$ & $0.307 \pm 0.043$ & $1.022 \pm 0.060^{\star \star \star}$ \\
\hline C-Ond & $0.284 \pm 0.238$ & $0.385 \pm 0.347$ & $0.047 \pm 0.021$ & $0.629 \pm 0.164^{\# \#}$ & $0.153 \pm 0.017$ & $0.306 \pm 0.038^{\# \#}$ \\
\hline C-XBXT & $0.235 \pm 0.126$ & $0.129 \pm 0.096$ & $0.008 \pm 0.006$ & $0.616 \pm 0.065^{\# \#}$ & $1.116 \pm 0.260^{\star \star \star \# \# \#}$ & $0.370 \pm 0.084^{\#}$ \\
\hline P-Model & $0.105 \pm 0.067$ & $0.050 \pm 0.039$ & $0.016 \pm 0.008$ & $0.868 \pm 0.073^{\star \star \star}$ & $0.378 \pm 0.126$ & $1.081 \pm 0.079^{\star \star \star}$ \\
\hline P-Ond & $0.379 \pm 0.284$ & $0.214 \pm 0.137$ & $0.028 \pm 0.010$ & $0.318 \pm 0.117$ & $0.110 \pm 0.047$ & $0.382 \pm 0.050^{\& \&}$ \\
\hline P-XBXT & $0.341 \pm 0.055$ & $0.235 \pm 0.174$ & $0.005 \pm 0.004$ & $0.116 \pm 0.022^{\& \&}$ & $0.152 \pm 0.036$ & $0.126 \pm 0.043^{\& \& \&}$ \\
\hline
\end{tabular}

${ }^{*} P<0.05,{ }^{* \star} P<0.01,{ }^{* \star} P<0.001$, compared with the Control; ${ }^{\#} P<0.05,{ }^{\# \#} P<0.01,{ }^{\# \# \#} P<0.001$, compared with the C-Model group; ${ }^{\&} P<0.05,{ }^{\&} \&<0.01,{ }^{\& \& \&} P<0.001$, compared with the P-Model group. Cisplatin (6mg/kg (weight), i.p.) and 1-PBG $(0.16 \mathrm{~g} / \mathrm{mL}$, i.p.) were administered at $\mathrm{t}=0$.

Table 2. Effects of XBXT on body weight of pica model rats $\square \pm S E M \square g \square$

\begin{tabular}{|lllllll|}
\hline & $-48 \mathrm{~h}$ & $-24 \mathrm{~h}$ & 0 & $24 \mathrm{~h}$ & $48 \mathrm{~h}$ & $72 \mathrm{~h}$ \\
\hline Control & $219.050 \pm 3.963$ & $227.767 \pm 5.037$ & $232.217 \pm 4.614$ & $234.950 \pm 5.087$ & $239.300 \pm 5.171$ & $244.067 \pm 5.840$ \\
\hline C-Model & $227.133 \pm 3.471$ & $233.517 \pm 3.731$ & $242.483 \pm 4.882$ & $232.250 \pm 4.681$ & $224.333 \pm 5.393$ & $217.267 \pm 5.433^{* * \star}$ \\
\hline C-Ond & $233.067 \pm 5.368$ & $223.000 \pm 3.887$ & $244.650 \pm 5.328$ & $244.667 \pm 3.976$ & $241.367 \pm 4.332$ & $228.783 \pm 4.831$ \\
\hline C-XBXT & $223.400 \pm 3.330$ & $231.900 \pm 3.887$ & $239.350 \pm 4.455$ & $233.183 \pm 4.720$ & $222.817 \pm 4.546$ & $216.000 \pm 5.5466^{* * \star}$ \\
\hline P-Model & $225.867 \pm 3.092$ & $234.233 \pm 2.828$ & $242.567 \pm 3.204$ & $244.350 \pm 3.122$ & $250.333 \pm 3.409$ & $259.183 \pm 3.966$ \\
\hline P-Ond & $220.150 \pm 2.666$ & $225.633 \pm 3.701$ & $232.350 \pm 3.562$ & $232.283 \pm 3.885$ & $236.900 \pm 3.902$ & $243.983 \pm 4.080$ \\
\hline P-XBXT & $219.617 \pm 3.543$ & $226.367 \pm 3.878$ & $233.317 \pm 4.362$ & $234.817 \pm 4.752$ & $238.867 \pm 6.550$ & $245.700 \pm 7.094$ \\
\hline
\end{tabular}


${ }^{*} P<0.05,{ }^{* *} P<0.01,{ }^{* * *} P<0.001$, compared with the Control; ${ }^{\#} P<0.05,{ }^{\# \#} P<0.01$, ${ }^{\# \# \#} P<0.001$, compared with the C-Model group; \& $P<0.05,{ }^{\&} \&<0.01,{ }^{\& \&} \&<0.001$, compared with the P-Modle group. Cisplatin (6mg/kg (weight), i.p.) and 1-PBG $(0.16 \mathrm{~g} / \mathrm{mL}$, i.p. $)$ were administered at $\mathrm{t}=0$.

Table 3. Diversity estimation of the $16 \mathrm{~S}$ rDNA gene library of the rats from the sequencing.

\begin{tabular}{|lllllll|}
\hline Groups & Total tags & OTUs & Chao & Ace & Shannon & Simpson \\
\hline Control & $89161 \pm 1390$ & $1457 \pm 25$ & $1901.61 \pm 22.85$ & $1975.86 \pm 20.37$ & $6.94 \pm 0.22$ & $0.96 \pm 0.01$ \\
\hline C-Model & $86599 \pm 1577$ & $1249 \pm 33$ & $1710.46 \pm 42.18$ & $1744.00 \pm 40.46$ & $6.31 \pm 0.32$ & $0.94 \pm 0.03$ \\
\hline C-Ond & $84087 \pm 937$ & $1102 \pm 32$ & $1456.76 \pm 52.42$ & $1496.53 \pm 58.78$ & $6.27 \pm 0.32$ & $0.95 \pm 0.02$ \\
\hline C-XBXT & $87630 \pm 1795$ & $1209 \pm 41$ & $1578.75 \pm 52.03$ & $1645.74 \pm 60.80$ & $5.85 \pm 0.40$ & $0.90 \pm 0.04$ \\
\hline P-Model & $81016 \pm 1139$ & $1209 \pm 27$ & $1615.70 \pm 38.02$ & $1655.24 \pm 34.14$ & $6.71 \pm 0.14$ & $0.97 \pm 0.01$ \\
\hline P-Ond & $90577 \pm 1972$ & $1189 \pm 42$ & $1593.96 \pm 73.45$ & $1626.17 \pm 73.20$ & $6.62 \pm 0.26$ & $0.96 \pm 0.01$ \\
\hline P-XBXT & $86091 \pm 1067$ & $1183 \pm 54$ & $1600.70 \pm 60.61$ & $1650.01 \pm 69.90$ & $6.44 \pm 0.28$ & $0.96 \pm 0.01$ \\
\hline
\end{tabular}

\section{Figures}


A

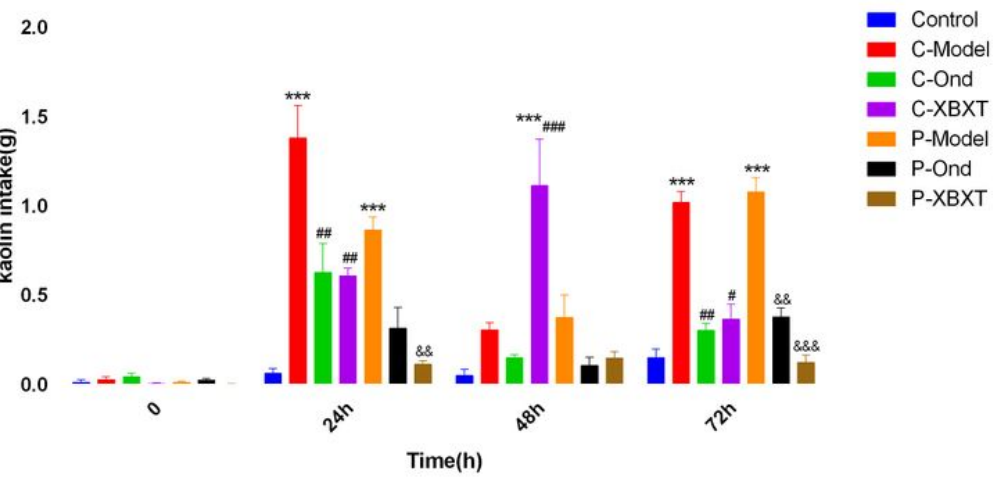

B
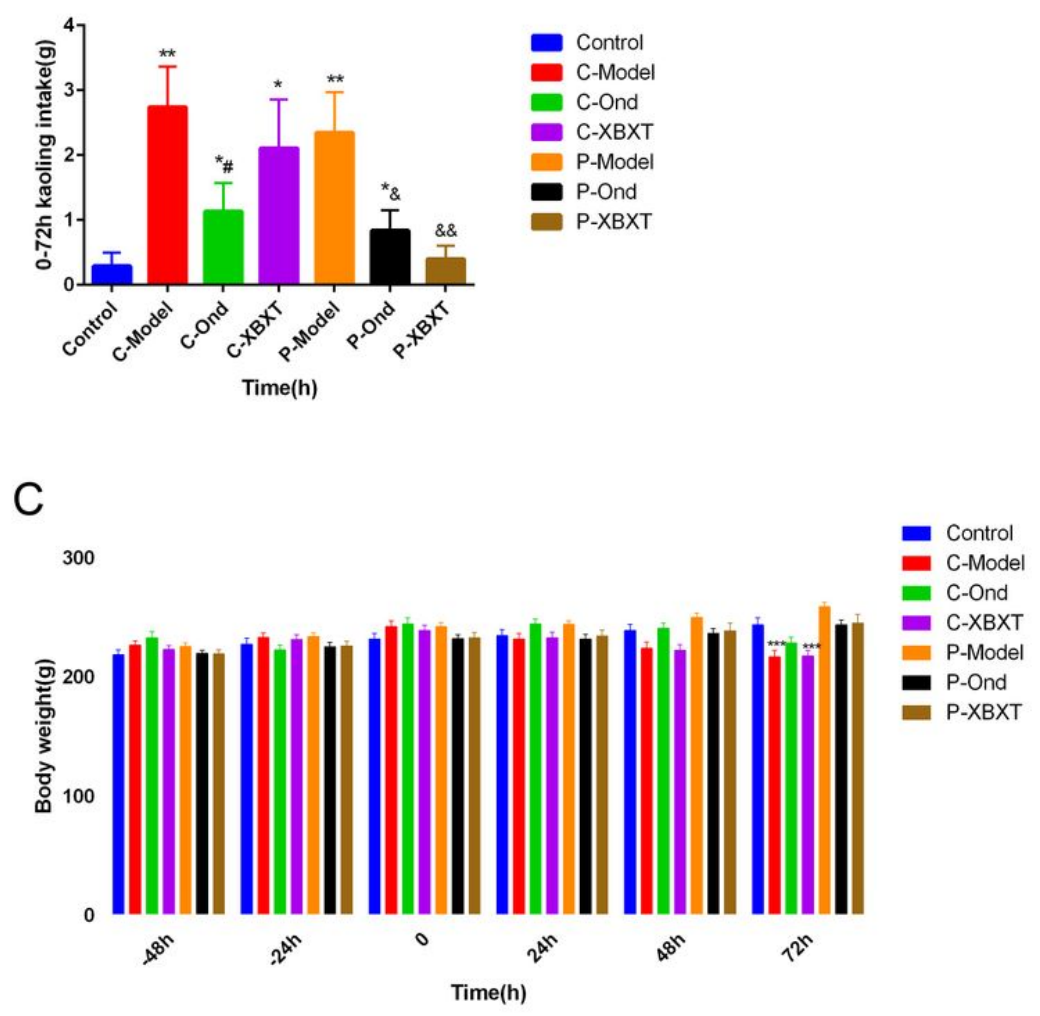

Figure 1

Effects of XBXT and ondansetron on kaolin consumption and body weight in cisplatin and 1-PBG treated rats. A: effects of XBXT and ondansetron on kaolin consumption at different time points; B: the total amount of kaolin intake of the 7 groups in 72 $\mathrm{h}$ after the injection of cisplatin and 1-PBG; C: effects of XBXT and ondansetron on body weight. ${ }^{*}<<0.05,{ }^{* \star P}<0.01,{ }^{\star \star *} \mathrm{P}<0.001$,

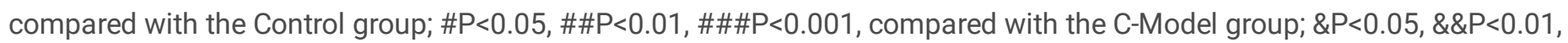
$\& \& \& P<0.001$, compared with the P-Model group. XBXT: Xiao-Ban-Xia-Tang; 1-PBG: 1- phenylbiguanide hydrochloride. 
A

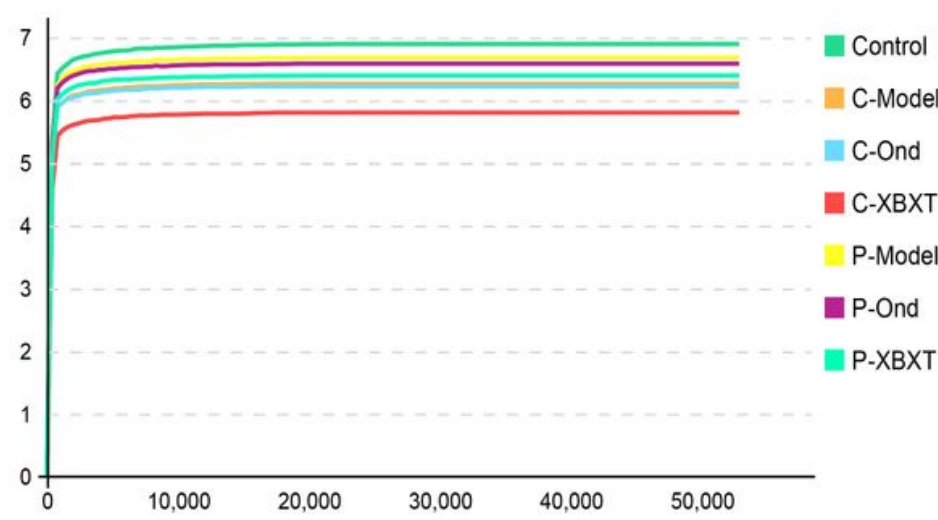

C

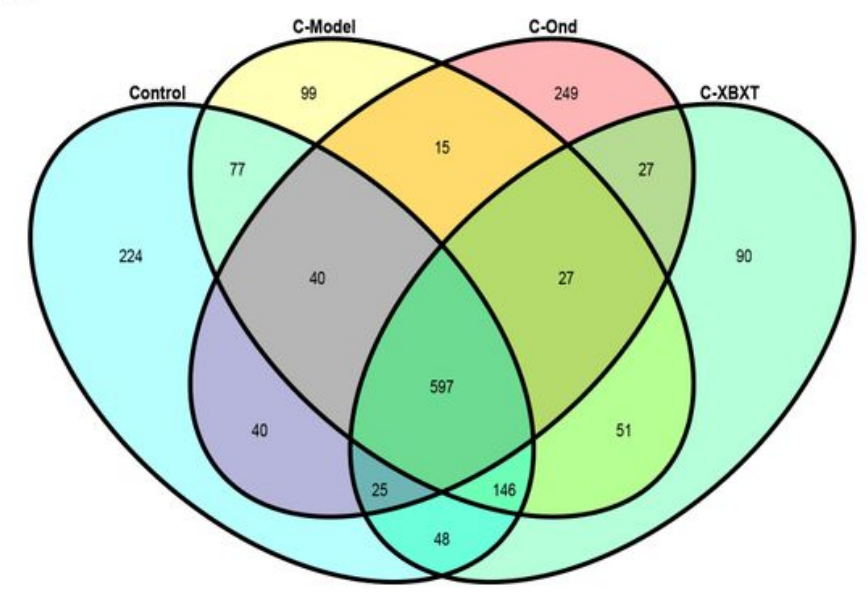

B

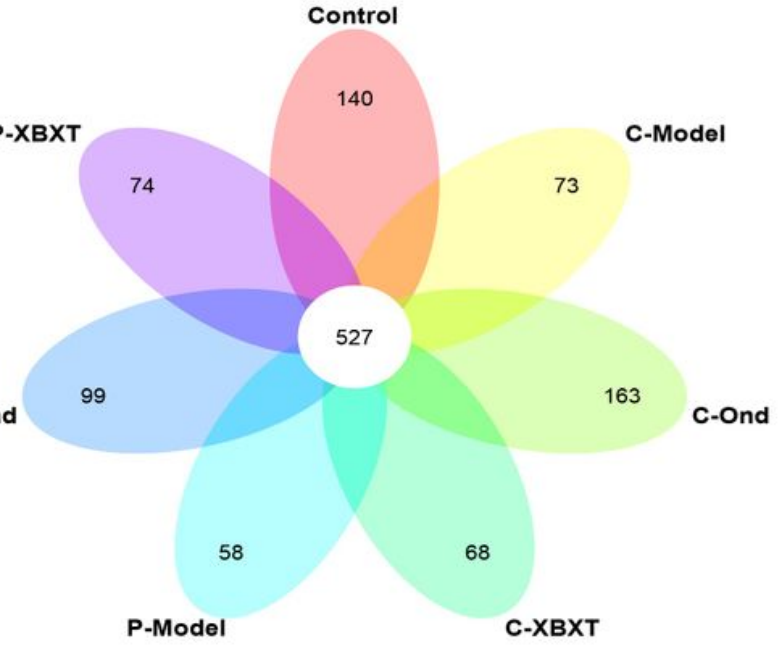

D

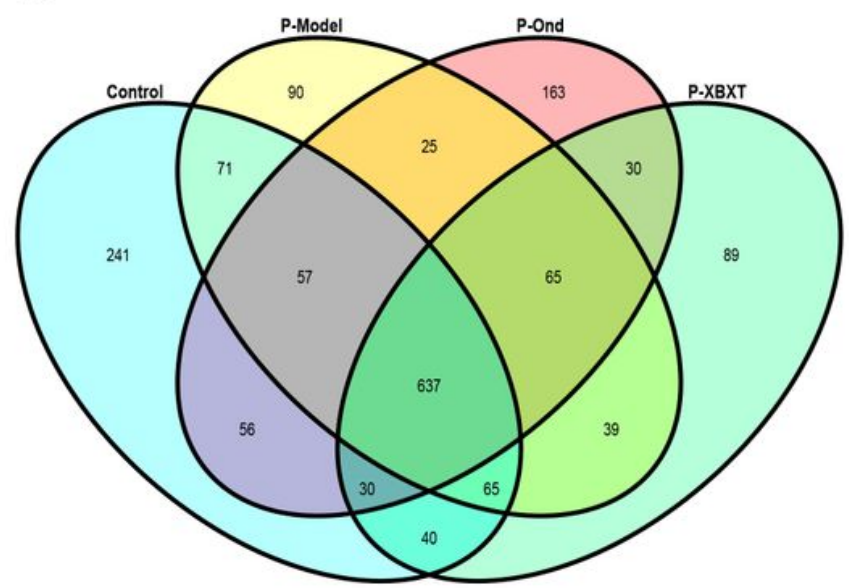

Figure 2

Overview of the $16 \mathrm{~S}$ rDNA gene analysis. A: the Shannon rarefaction curves for each group; B: the distribution of OTUs in the 7 groups of rats; C: the distribution of OTUs in the cisplatin treated rats; D: the distribution of OTUs in the 1-PBG treated rats. 1PBG: 1- phenylbiguanide hydrochloride. 
A

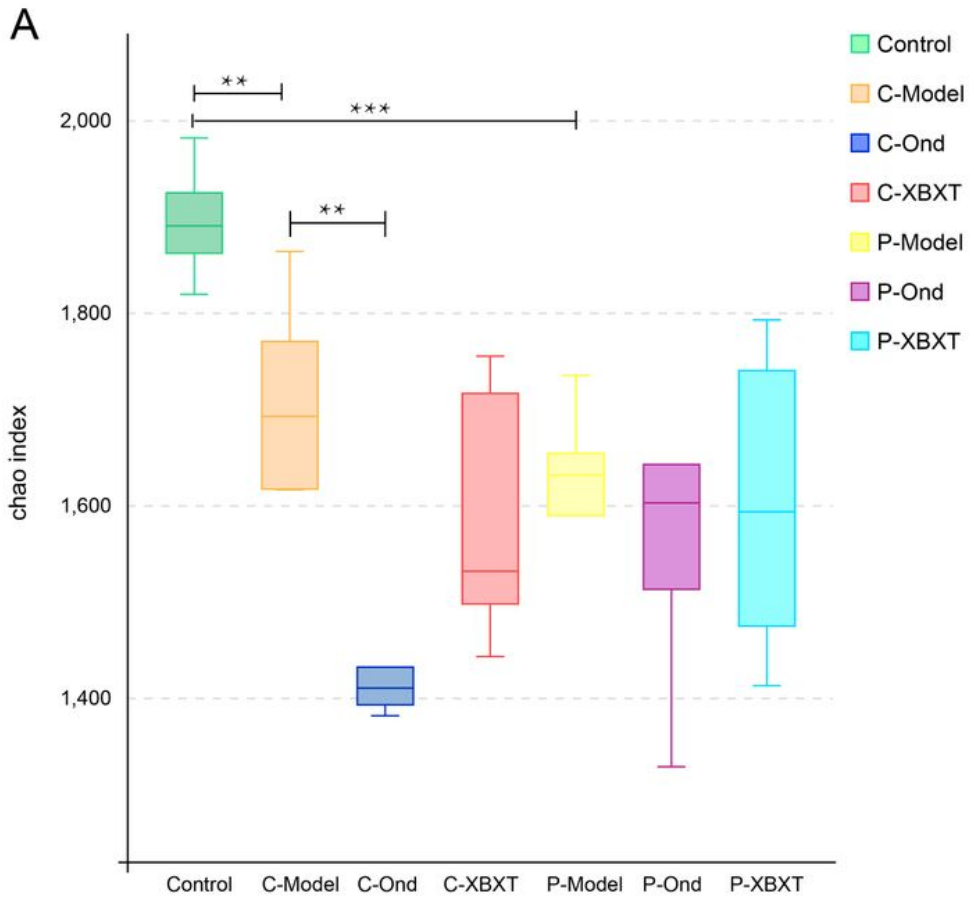

B

PCoA

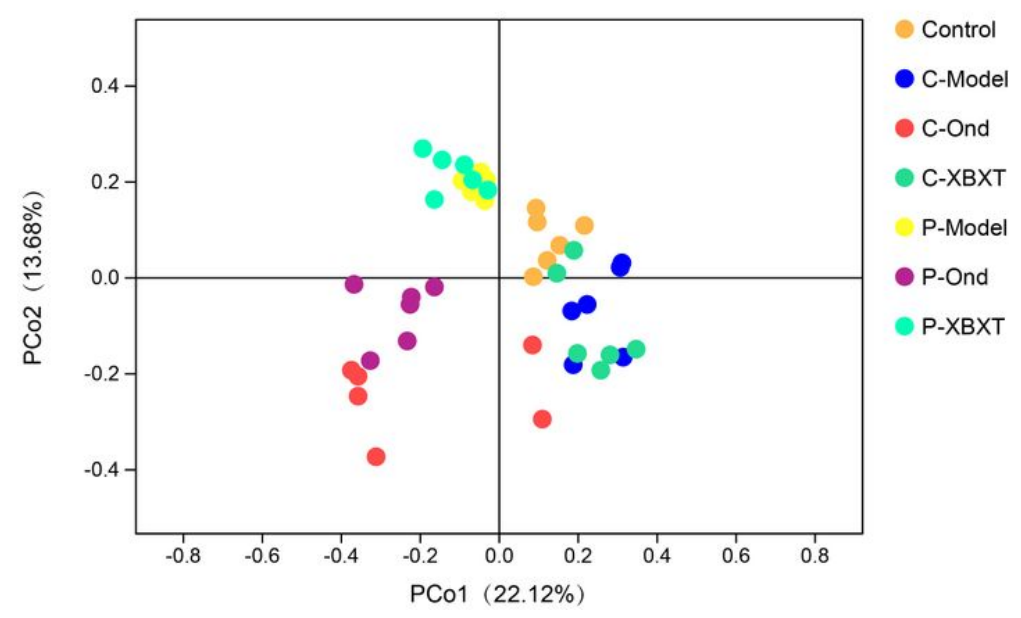

Figure 3

The alpha diversity and the Unifrac analyses of the gut microbiota in the 7 groups. A: the alpha diversity of the 7 groups; $\mathrm{B}$ : the Principal co-ordinates analysis (PCOA) of the 7 groups of rats. 


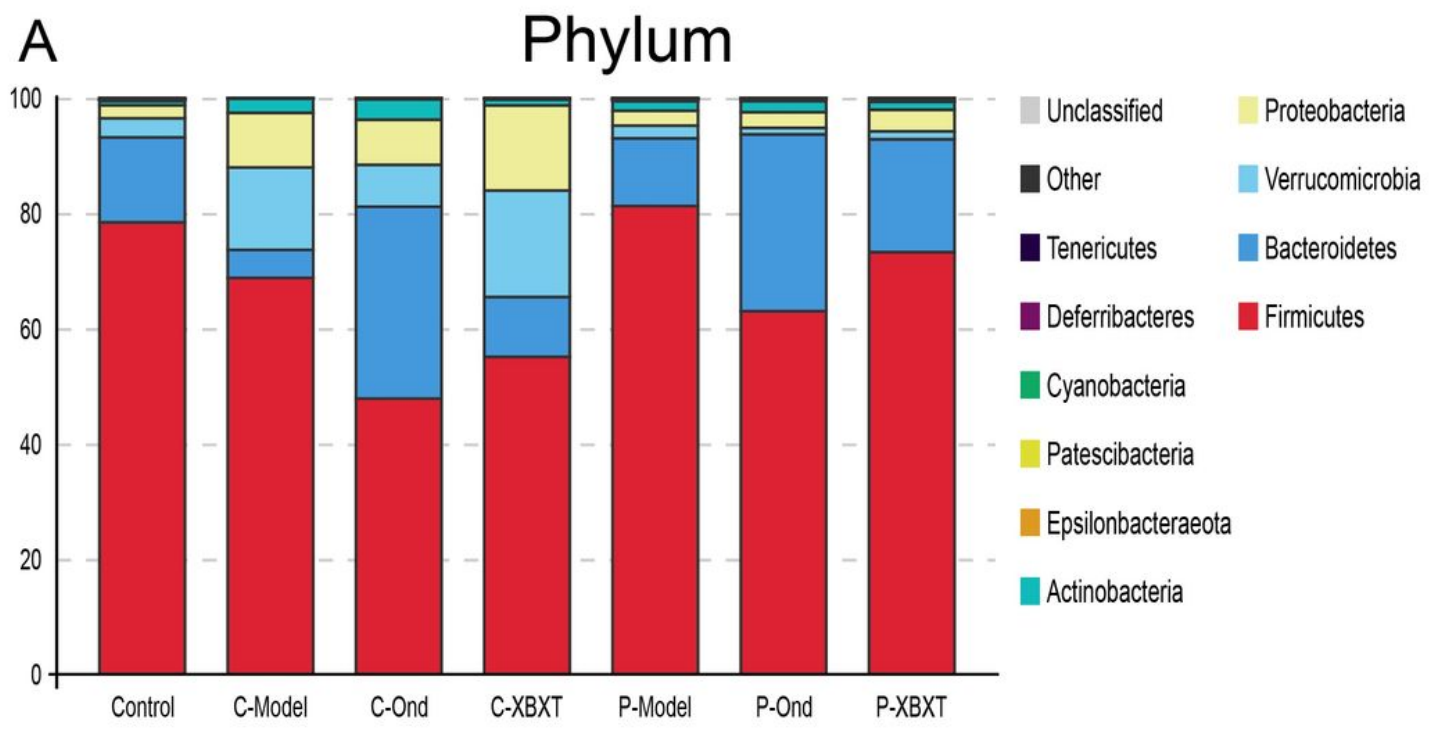

B

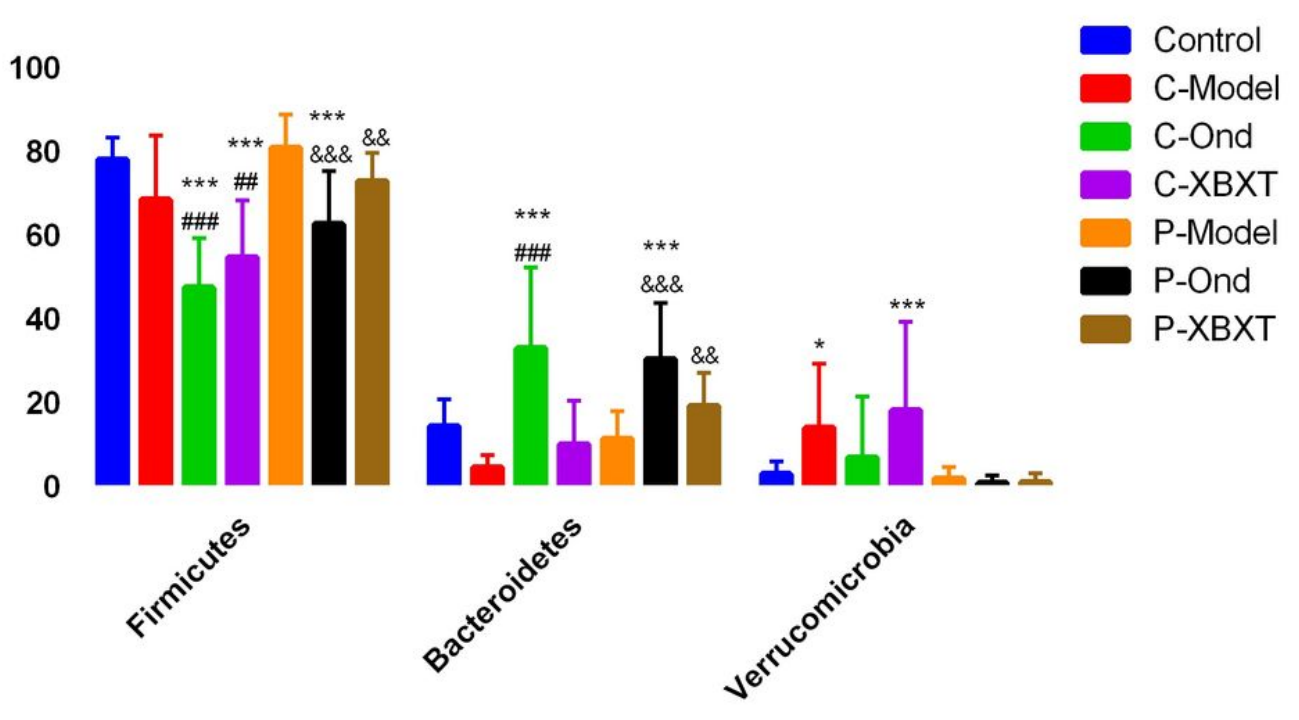

Figure 4

The relative composition and abundance of the gut microbiota at the bacterial phylum level. A, B: the relative abundance of the gut microbiota of the 7 groups of rats at the bacterial phylum level. 

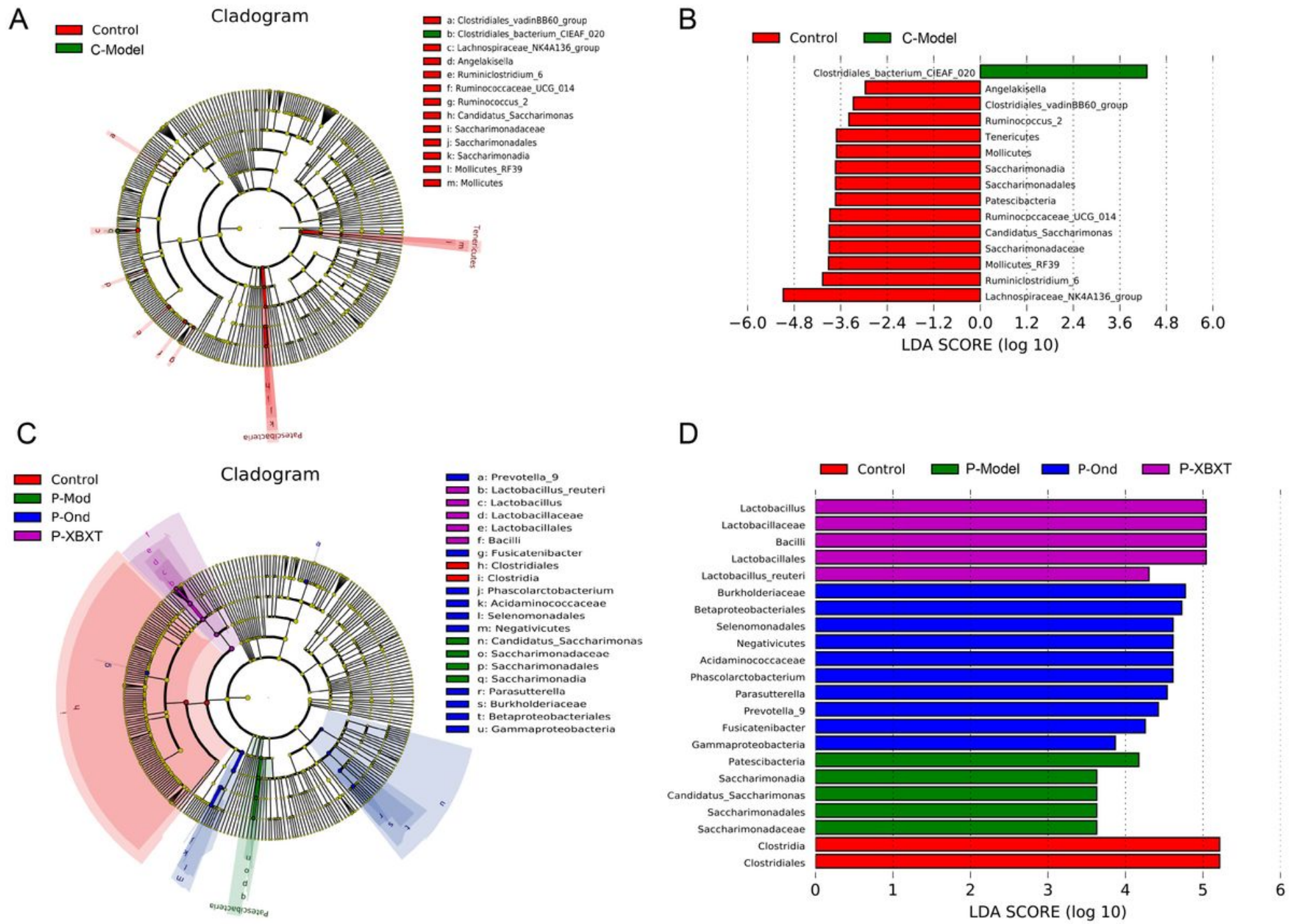

\section{Figure 5}

LEFse analysis of the cisplatin treated groups and the 1-PBG treated groups. A: LEFse analysis identifed the predominant taxons of the cisplatin treated groups; B: LEFse analysis identifed the predominant taxons of the 1-PBG treated groups. 1-PBG: 1- phenylbiguanide hydrochloride.

\section{Supplementary Files}

This is a list of supplementary files associated with this preprint. Click to download.

- SupplementalFigure4.tif

- SupplementalFigure3.tif

- SupplementalFigure2.tif

- SupplementalFigure1.tif 